for producing quality controlled steels and alloys in fairly high tonnages. It is economically competitive with other processes at present used, as the capital outlay is fairly low and the running cost is the same as if not cheaper than the other processes.

\section{Shocks in Store}

THe bid by the General Electric Company for Associated Electrical Industries seems to have taken AEI by surprise. AEI is taking a week to think about the bid, worth $£ 120$ million in stock and cash, before committing itself to a view. GEC has offered five GEC shares and $80 s$. cash for every eight AEI shares, which effectively puts a value of $52 s$. $1 d$. on the AEI shares against a market price before the bid of $43 s .6 d$. By October 3, AEI shares stood at 56s. 4.5d., as high as they have been this year, indicating a feeling among investors that AEI will offer at least some opposition to the takeover. The terms, AEI is likely to claim, are based on a poor performance by AEI last year, and a forecast of profits of about $£ 9.2$ million this year. It is likely to add that better times are on the way, but shareholders have been told this many times before. GEC profits are far more buoyant, running at about twice those of AEI, although GEC is a much smaller group.

Both companies employ large research departments. AEI has a large central research laboratory at Rugby, which carries out basic research for the whole company as well as contract research work for government and other companies. AEI Power Group has a laboratory at Trafford Park in Manchester which includes among its interests scientific instruments, traction and magnetohydrodynamic power generation. The Telecommunications Group runs two laboratories, one at Blackheath and one at Harlow, and the Cables Group has a laboratory at Gravesend concerned with insulating materials, and one at Woolwich studying high voltage phenomena. The joint AEI-Thorn semiconductor company also runs a research department.

GEC's main laboratory is the Hirst Research Centre in North Wembley, named after Sir Hugo Hirst, the founder of the company. This has divisions serving all branches of the business-lamps and lighting, telecommunications, electronics, valves and semiconductors. It also includes three divisions doing more fundamental work, in engineering, metallurgy and electronics materials, and a division which supplies chemical and technical services. In addition, GEC Electronics runs two applied electronics laboratories, at Stanmore and Portsmouth, and there is another telecommunications laboratory at Coventry. The Osram lamp company has two laboratories, and other companies associated with GEC also run their own development laboratories.

If the takeover goes through, some rationalization of this mass of laboratories seems inevitable. This is particularly likely in electronics, where the new group would hold 40 per cent of the market. GEC has a joint semiconductor company with Mullard, while AEI shares its electronics interests with Thorn; if the deal is successful, GEC hopes to merge these interests and, save money on research. It is not yet clear how the deal would affect AEI's nuclear power interests-it owns 10 per cent of the shares of the Nuclear Power Group, Ltd. GEC's association with nuclear power ended some years ago, and was not a happy one. GEC was involved in the building of an unsuccessful power station in Japan, and still has problems to solve at the Hunterston $A$ station which it built. AEI's nuclear power interests have certainly been more profitable than this, but whether this would encourage GEC to go back into nuclear power as part of the Nuclear Power Group, or pull out altogether, is still not clear.

\section{Laboratory of All Trades}

SEWAGE is unattractive but inescapable. At the Water Pollution Research Laboratory, the staff continues to study it with every evidence of enthusiasm, and this year is spending $£ 360,000$ both on the treatment of sewage and on its effects when allowed to escape. A scheme in collaboration with the Hydraulics Research Station and a firm of consulting engineers is intended to find the ideal position for sewage outflow in the Thames Estuary. In parallel, the laboratory is studying the effect of pollution on fish, together with the related problems of the variation and measurement of oxygen concentration in streams and rivers.

The laboratory has been hard at work on the two chief methods of biological treatment--the activated sludge process and biological filtration. One interesting development is the use of plastic filters in biological filtration, and a system has been devised for controlling automatically the intensity of aeration so as to maintain oxygen at an optimum concentration. A new treatment of sewage, by chemical oxidation at high temperatures and pressures, is undergoing preliminary investigation, and as a means of reaching high pressures it has been suggested that the sewage be poured into very deep holes in the ground. The laboratory is optimistic about the change from hard to soft detergents; this, it says, has had the effect of markedly reducing the amount of foam in rivers. The manufacturers and the laboratory are hoping to find even softer detergents, and are conducting an experiment at a small sewage works serving a village community specially supplied with experimental detergent by the manufacturers.

Pollutants are also studied at the Warren Spring Laboratory, but in this case air is the dispersion medium. The laboratory provided background information for the Clean Air Act and is at present completing a five-year national survey of smoke and sulphur dioxide. Smoke concentration in the air has been falling, and although total emission of sulphur dioxide has risen slightly, the overall concentration of the gas has fallen.

Recovery of metals from scrap, studies of process control and separation of dry minerals by air fluidization form an important part of the work of the mineral science and technology section and the chemical engineering section. Both work in consultation with industry. The chemical engineering section has considerable interest in catalysts. A pilot plant producing acetone by dehydrogenation of isopropanol has been linked to a computer for process control studies, but this system has limited application. Control by simple logic systems of batch processes such as distillation of binary mixtures is being studied and is likely to be more useful. The laboratory employs a staff of 440 , and is expanding slightly year by year, with annual expenditure standing currently at $£ 1$ million. 